\title{
SVASTA, PRANA and VaU: Three Novel Ventilators from Space Community
}

\author{
Design and Development Team ${ }^{1}$
}

Received: 19 May 2020 / Revised: 15 June 2020 / Accepted: 28 June 2020 / Published online: 23 July 2020

(C) Indian National Academy of Engineering 2020

\begin{abstract}
This paper describes the design and development of three low-cost ventilators which can be mass produced to meet a surge in demand for such equipment. Each of these ventilators is having unique features while meeting standard functional specifications of mechanical ventilators. The first design works on compressed air source as control gas and does not require electrical power for its operation. Parameters like tidal volume, breathing rate etc. can be set mechanically in this ventilator. Second one is an automated Ambu bag based system, which has digital controller providing closed-loop control of critical ventilator parameters and a very unique geared actuation system that ensure long cyclic life to Ambu bag and fine control of parameters. The third design is an air blower based pneumatic circuit ventilator which is a state of the art ICU ventilator. Mathematical modeling of the systems is carried out to design the mechanical/electrical control elements and to simulate the performance of the system. Prototypes of ventilators were developed, testing and simulation runs were carried out and critical parameters were measured ensuring satisfactory performance of the system.
\end{abstract}

Keywords Ventilator $\cdot$ Compressed air $\cdot$ Bag valve mask $(B V M) \cdot$ Mathematical modeling $\cdot$ Testing

\section{Introduction}

COVID-19, an infectious disease caused by novel coronavirus is by far a very severe respiratory illness causing the disease which has affected nearly 3 million people worldwide and caused nearly 0.2 million deaths as on $30^{\text {th }}$ April 2020 (05:30 GMT) as per the report of World Health Organization (WHO) (2020). It has also been declared as a pandemic disease now spread to nearly 213 countries, areas and territories. In India, there are $~ 24,000$ confirmed cases and $\sim 1000$ deaths caused by this infectious disease as on 30th April 2020 (Website of Ministry of Health and Family Welfare 2020). An overwhelming number of COVID-19 patients are getting admitted to Intensive Care Units (ICUs) and a large number of them require respiratory support through mechanical ventilation.

In the event of an uncontrolled spread of the disease, the demand for mechanical ventilators is likely to increase.
Hence, low-cost mechanical ventilators are the need of the hour to aid the COVID-19 victims.

Mechanical ventilators are machines which mechanically assist the patient's breath. Ventilator is typically connected to the patient through the breathing circuit as shown in Fig. 1.

Vikram Sarabhai Space Centre (VSSC) is developing three models of a mechanical ventilator, a control chamber based design named 'Space Ventilator Aided System for Trauma Assistance' (SVASTA), an Ambu bag (A commercial manual respirator bag) based design named 'Programmable Respiratory Assistance for the Needy Aid' (PRANA), and a pneumatic circuit based system named 'Ventilation assist Unit' (VaU). VaU is developed with support from other ISRO centres. SVASTA is a novel piston ventilator, PRANA is an Ambu bag based ventilator and VaU is a turbine ventilator.

Design and Development Team

victor_joseph@vssc.gov.in

1 Vikram Sarabhai Space Centre, Thiruvananthapuram, India 


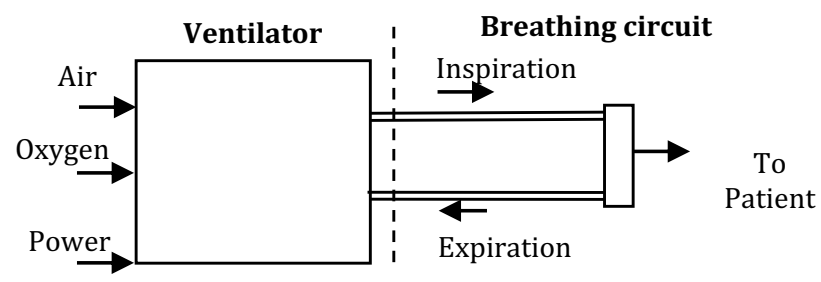

Fig. 1 Schematic of Mechanical Ventilator

\section{Types of Mechanical Ventilators and Recent trends}

Evolution of the concept of mechanical ventilation can be summarized using Fig. 2. The initial mechanical ventilators were not equipped with sensors (Fig. 2a), and later progressed to monitor the ventilation parameters, allowed closed-loop control and provided critical information to the clinicians (Fig. 2b). The latest ones are those which monitor the health of the patients and adaptively adjust the ventilator parameters on the basis of patients' needs (Fig. 2c).

Mechanical ventilators are typically powered by electricity or compressed gas. Based on the method of generating gas flow, mechanical ventilators can be divided as follows (Methods of generating gas flow for mechanical ventilation 2020):

- Pneumatic ventilators

Vast majority of ventilators used in hospitals belong to this class. These ventilators use compressed gas and converts them to the required pressure and can achieve very high flow rate (Fig. 3a). Solenoid valves are generally used as the control element which releases pressurized gas into a gas mixing chamber.

- Turbine ventilators

These ventilators generate flow by means of a turbine referred to as a "blower" (Fig. 3b). Room air is sucked into the ventilator by means of the negative pressure generated by the blower. Upstream from the blower, fresh oxygen gas is added to the circuit. The turbine compresses the gas mixture and generates gas flow.
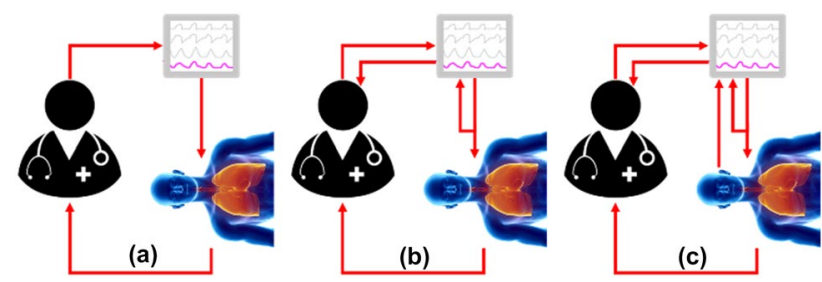

Fig. 2 Evolution of Mechanical Ventilators (Dellaca' et al. 2017)

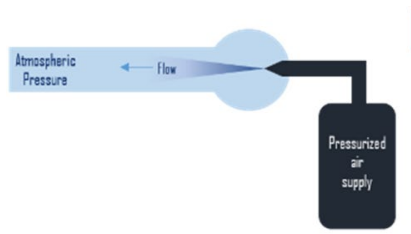

(a)

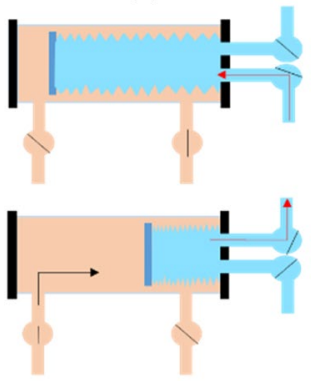

(c)

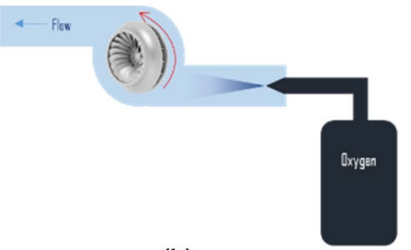

(b)
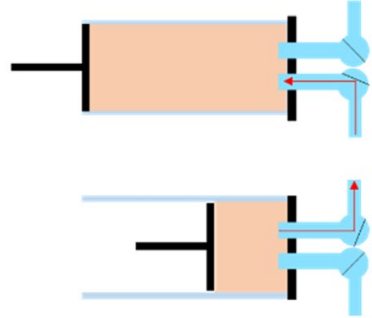

(d)
Fig. 3 a Pneumatic ventilator (b) Turbine ventilator (c) Bellows ventilator (d) Piston ventilator (2020)

- Double Circuit-Bellows ventilators

"Bellows" is a reservoir which stores the gas mixture which is supplied to the patient. By applying external pressure to this reservoir, inspiratory flow is generated (Fig. 3c).

- Single Circuit-Piston ventilators

Here, unlike bellow ventilators, a plunger pulls and pushes the fresh gas inside a cylinder and generates the gas supply (Fig. 3d).

Owing to the sudden increase in demand for ventilators due to COVID-19 pandemic, hardware enthusiasts, academics, industries, etc. are coming forward with easy-to-build high-pressure ventilator tailored specifically to treat COVID19 patients. Most notable developments during these period are the following:

a. NASA engineers have developed a high-pressure ventilator called VITAL (Ventilator Intervention Technology Accessible Locally), a mechanism that's controlled by embedded electronics (NASA Develops COVID-19 Prototype Ventilator in 37 Days2020).

b. IIT Roorkee: A low-cost portable ventilator called 'Prana-Vayu', working in closed-loop is developed in collaboration with AIIMS, Rishikesh (Eight Indian Innovation 2020).

c. Indian Railways has manufactured a low-cost ventilator prototype named 'Jeevan' which is production friendly as it uses smaller size components or locally sourced components (Indian Railways Manufactures 2020).

d. MIT Emergency Ventilator (E-Vent) Project: A team of MIT Engineers and American clinicians have generated 
an open-source design based on automating a manual resuscitator.

\section{Requirements of ventilator}

Mechanical ventilators assist or replace spontaneous breathing when a patient's own breathing is not sufficient. Typical requirements of a mechanical ventilator are given below; the values for parameters are taken from references (Medicines and Healthcare 2020) and (World Health Organization (WHO) 2020):

a. Mixes oxygen and air to achieve the required inspired oxygen concentration $\left(\mathrm{FiO}_{2}\right)$ set by the user $(30 \%-100 \%$, typically $40 \%$ ). In the envisaged scenario of a shortage of oxygen/medical air, the ventilator is designed to work with atmospheric air.

b. Delivers the required volume (specified by the user as tidal volume) of the gas mixture to the patient during inspiration (in volume-controlled ventilation).

c. Delivers the mandatory breaths per minute specified by the user.

d. Controls the expiratory valve setting (specified by the user) to maintain a minimum positive pressure in the breathing circuit throughout ventilation. (Positive End Expiratory Pressure (PEEP) recorded at end of expiration)

e. Supports 3 basic modes of ventilation namely Volume Control Ventilation (VCV) where the volume delivered per breath is controlled, Pressure Control Ventilation (PCV) where the inspiratory pressure is maintained constant and Pressure Support Ventilation (PSV) where inspiration is triggered based on patient's attempt to breath

f. Detects inspiratory attempts from the patient (based on breathing circuit pressure momentarily going below
PEEP) and initiates/supports the delivery of gas to lungs. This is required for Pressure Support Ventilation (PSV) or Synchronized intermittent mandatory ventilation (SMIV).

g. Records inspiratory and expiratory flow, pressure \& volume. Provides a graphical display of the same for analysis by the user to assess patient health. Bluetooth interface and android app may be developed for data transfer and plotting. This may be required if large numbers of ventilators are to be monitored simultaneously by available staff. Essential parameters can be displayed in the ventilator.

h. General specifications of mechanical ventilators are Tidal volume setting in the range $200-600 \mathrm{ml}$ in steps of $50 \mathrm{ml}$, Inspiration pressure up to $70 \mathrm{~cm}$ of water, Respiratory Rate of 10-30 breaths per minutes in increments of 2, Inspiratory: Expiratory ratio (I:E) in the range of 1:1 to $1: 3$ and Fraction of inspired oxygen $\left(\mathrm{FiO}_{2}\right)$ between 30 to $100 \%$ in $10 \%$ steps.

Comparison of specifications \& requirements from the references and those adopted for the ventilators being developed is shown in Table 1.

\section{Design Options for Mechanical Ventilator}

\section{SVASTA}

This novel ventilator is designed to work without electric power. The system operates on compressed air and achieves different modes of ventilation through mechanical settings alone. Electronics is proposed for displaying sensor data and for On/Off control of solenoid valves.
Table 1 Comparison of ventilator parameters

\begin{tabular}{|c|c|c|c|}
\hline Parameter & $\begin{array}{l}\text { Ref ( Medicines and } \\
\text { Healthcare 2020) }\end{array}$ & $\begin{array}{l}\text { Ref (World Health } \\
\text { Organization } \\
\text { (WHO) 2020) }\end{array}$ & SVASTA, PRANA, VaU \\
\hline $\mathrm{FiO}_{2}(\%)$ & $30-100$ & $21-100$ & $30-100$ \\
\hline Modes & Must have CMV & $\begin{array}{l}\text { PRVC PCV, VCV, } \\
\text { SIMV, PSV, non- } \\
\text { invasive }\end{array}$ & PCV, VCV, PSV \\
\hline Tidal volume (ml) & $350,400 \pm 10,450$ & $20-2000$ & $200-600$ \\
\hline Inspiration pressure $\left(\mathrm{cmH}_{2} \mathrm{O}\right)$ & $15-40$ & $0-40$ & Up to 70 \\
\hline Respiratory rate (breath per min) & $10-30$ & $10-60$ & 10 to 30 \\
\hline$I: E$ ratio & $1: 1-1: 3$ & IE and inverse IE & $1: 1-1: 3$ \\
\hline
\end{tabular}

$C M V$ Continuous Mandatory Ventilation, $P R V C$ Pressure Regulated Volume Control, $P C V$ Pressure Control Ventilation, $V C V$ Volume Control Ventilation, SIMV Synchronised Intermittent Mandatory Ventilation, PSV Pressure Support Ventilation 


\section{Configuration}

Configuration of the system is highlighted in Fig. 4. The sub systems of SVASTA are configured in three decks with the heart of the system, the volume control chamber/respirator, placed in the top deck along with system controller and display. The middle deck consists of an Air/oxygen blender connected to the input line for manual control of $\mathrm{FiO}_{2}$, and a humidifier connected to the Inspiratory line for humidification of gases delivered to the patient. The bottom deck consists of the expiration unit along with the PEEP monitoring system.

\section{Working}

The schematic of the volume control chamber and hardware is shown in Fig. 5. It consists of a respirator chamber and a control chamber with a common piston having piston heads in both chambers. Forward and reverse movements of the piston causes respiratory gases to be sucked into the respiratory chamber and delivered to the patient through a set of mechanical non-return valves.

The direction of movement of the piston is controlled by the position of switching valves located in the piston head of the control chamber. The valve pair directs the inlet control air to one side of the control chamber and simultaneously connects the other side of the control chamber to the vent port. The valve pair automatically switches its position when the piston reaches one end which in turn reverses the direction of motion of the piston. When control air is admitted to the system, the piston reciprocates continuously, delivering one breath in each cycle. Orifice pairs are put in inlet and vent lines, the size of which controls the duration of forward and return stroke of the piston, in turn controlling the $I: E$ ratio of the ventilator.

The breath per minute is controlled by the absolute value of the control pressure, an increase in control pressure

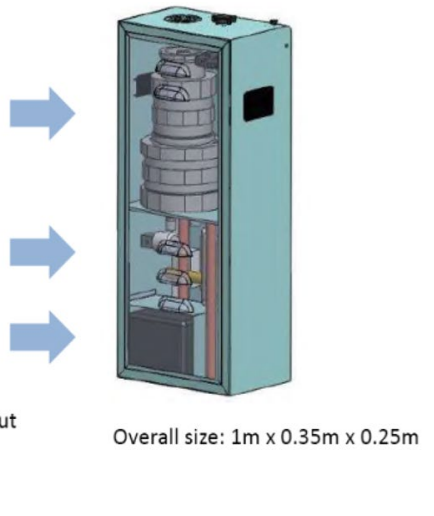

- Medical air supply in

- 02 supply in

- Ventilator outlet

Fig. 4 Configuration of SVASTA
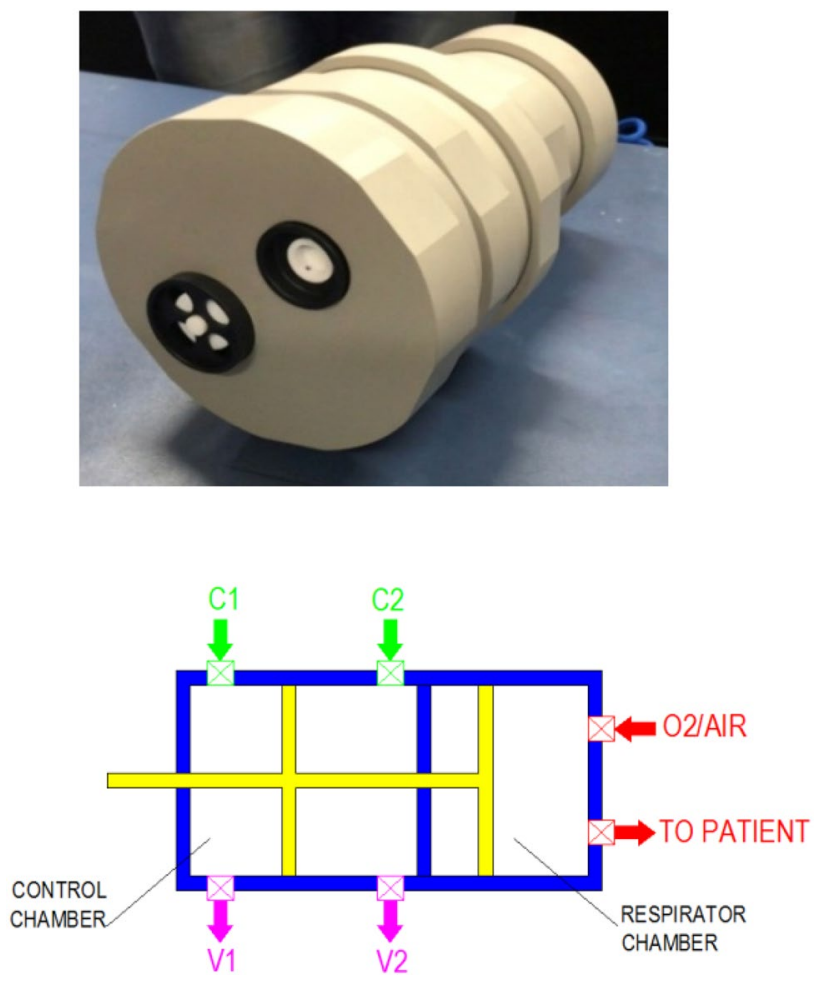

Fig. 5 Volume control chamber

increasing the breath per minute. The tidal volume delivered per breath depends on the length of the control chamber. This is also adjustable manually by rotating the knob to axially move the endplate of the control chamber.

The circuit diagram of the system is shown in Fig. 6 . Compressed air is admitted into the volume chamber through a pressure regulator for control. A novel mechanical orifice controller is incorporated in this line for manually selecting orifices to adjust the I:E ratio. The volume chamber can take in respiratory gases from air/oxygen blender or from atmosphere and deliver to the patient through humidifier. The air

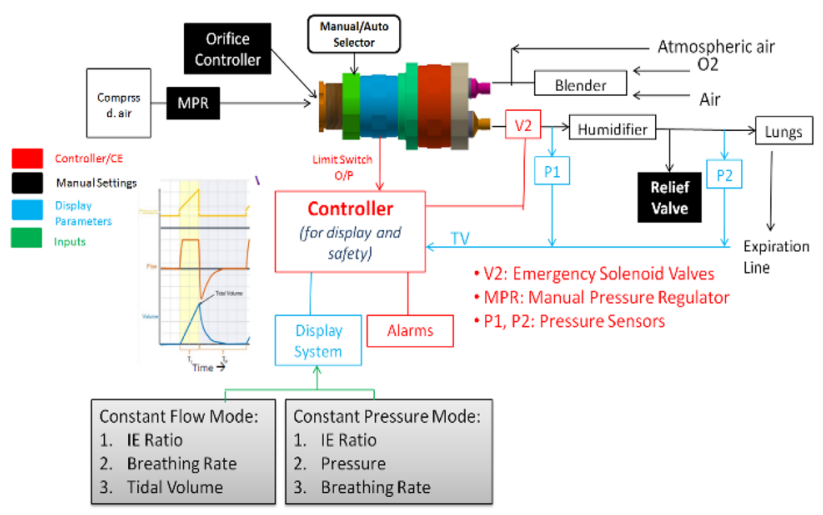

Fig. 6 Circuit diagram of "SVASTA" 
oxygen blender can mechanically set the $\mathrm{FiO}_{2}$ by medical air and oxygen in the required proportion. The system controller acquires pressure sensor data and displays flow, pressure and volume. The controller can also raise alarms and close the solenoid valve in case of an emergency.

\section{Mathematical modeling}

A mathematical model is developed from fundamental principles coupling mass flow rate equation, ideal gas equation and force equilibrium equation as given below. A linear lung resistance is assumed in the model. The typical waveforms for piston velocity, respirator pressure and mass flow rate from the ventilator for volume-controlled ventilation is shown in Fig. 7.

Elementary equation of mass flow through an orifice is given by Eq. (1).

$\dot{m}=C_{d} A_{O} P_{u} \sqrt{\frac{2}{R T}\left(\frac{\gamma}{\gamma-1}\right)\left(r^{\frac{2}{\gamma}}-r^{\left(\frac{\gamma+1}{\gamma}\right)}\right)}$

where $C_{d}, A_{O}, P_{u}, R, T$ and $\gamma$ represent the coefficient of discharge of orifice, orifice area, upstream pressure, ideal gas constant, gas temperature and specific heat ratio, respectively. $r$ is defined as the ratio of downstream pressure and upstream pressure. Below critical pressure ratio, the flow of gas gets chocked. Conditions for the same is given in Eq. (2).

$r= \begin{cases}\frac{P_{d}}{P_{u}} & 1 \geq r \geq\left(\frac{2}{\gamma+1}\right)^{\frac{\gamma}{\gamma-1}} \\ \left(\frac{2}{\gamma+1}\right)^{\frac{\gamma}{\gamma-1}} & r<\left(\frac{2}{\gamma+1}\right)^{\frac{\gamma}{\gamma-1}}\end{cases}$

For air $(\gamma=1.4)$, the critical pressure ratio is 0.528 .

Differential equation of motion of the piston is given in Eq. (3).

$M \ddot{x}+C \dot{x}=\sum F_{e x}$

where $M, C, F_{e x}$ and $x$ are mass of the piston, coefficient of viscous friction, external force acting on the system, and displacement of piston respectively.

Pressure inside the chamber is derived from energy (Eq. 4) and ideal gas (Eq. 5) (Richer and Hurmuzlu 2000) and given in Eq. (6).

$q_{\text {in }}-q_{\text {out }}+\gamma C_{v}\left(\dot{m}_{\text {in }} T_{\text {in }}-\dot{m}_{\text {out }} T\right)-\dot{W}=\dot{U}$

$P=\rho R T$

where $q_{\text {in }}, q_{\text {out }}, \gamma, C_{v}, \dot{m}_{\text {in }}, \dot{m}_{\text {out }}, T_{i n}, T, \dot{W}, \dot{U}, \mathrm{P}, \rho$ and $R$ are heat coming into the chamber, heat going out to the chamber, specific heat ratio, specific heat at constant volume, mass flow coming into the chamber, mass flow going out from

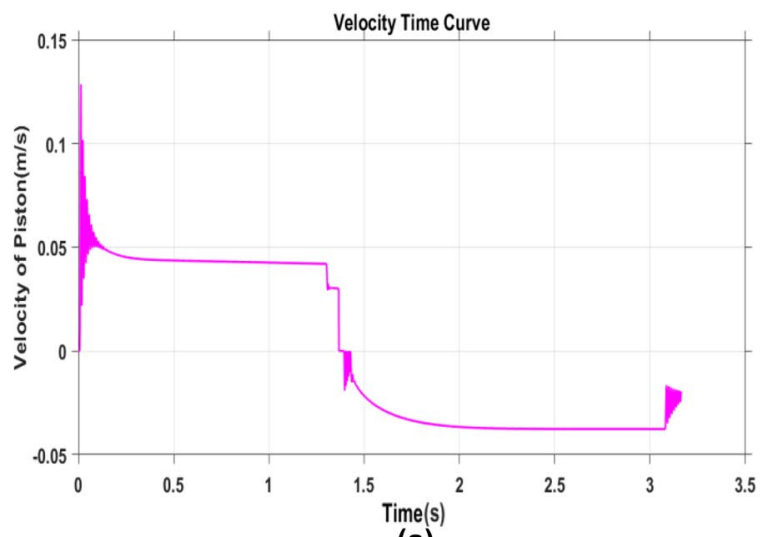

(a)

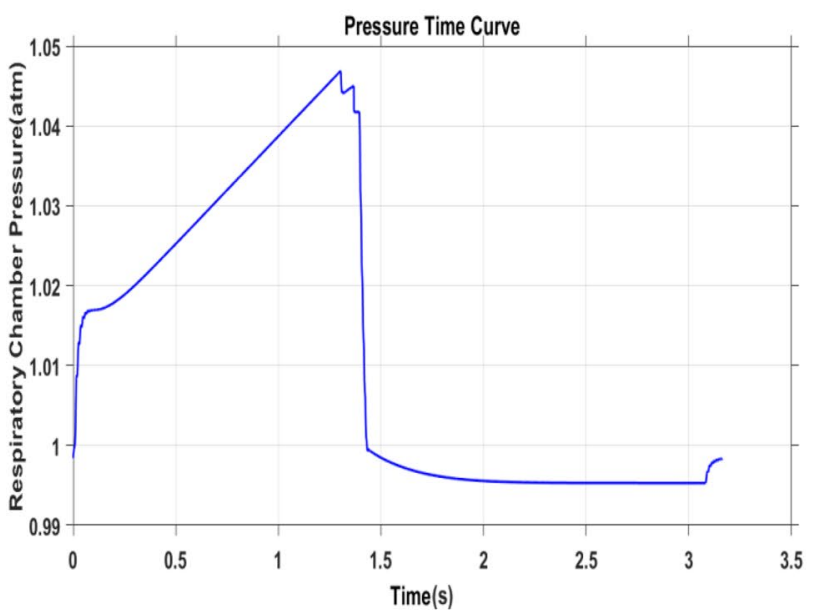

(b)

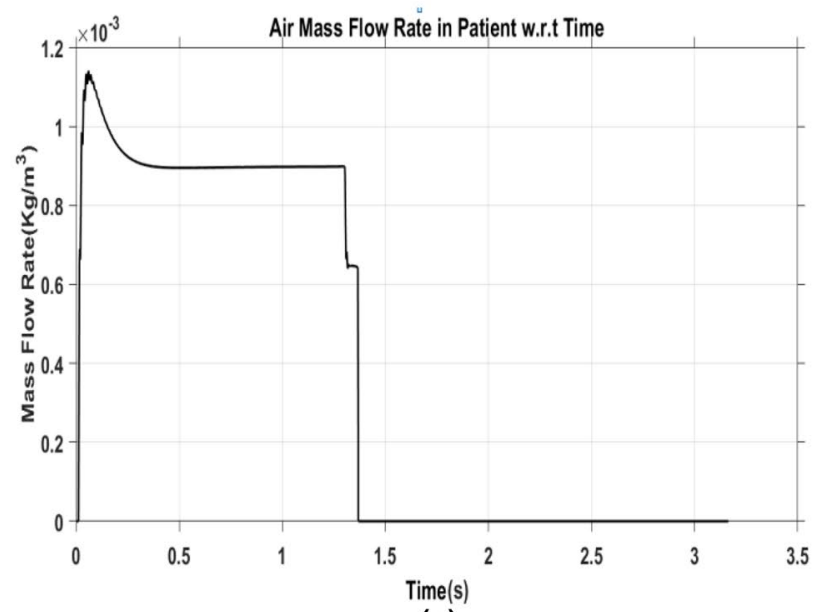

(c)

Fig. 7 Results from mathematical model for Volume Control Ventilation mode (a) Velocity of piston vs Time (b) Respiratory Piston Chamber pressure vs Time (c) Mass flow rate vs Time

the chamber, temperature of gas coming into the chamber, temperature of gas inside the chamber, work done rate, rate of change of internal energy, pressure of gas inside the chamber, density of gas and ideal gas constant respectively. 
Derivation of the rate of change of pressure, given in Eq. 6, inside the chamber of the double actuating piston is given in reference (Richer and Hurmuzlu 2000).

$\dot{P}=\frac{R T}{V}\left(\alpha_{\text {in }} \dot{m}_{\text {in }}-\alpha_{\text {out }} \dot{m}_{\text {out }}\right)-\alpha \frac{P \dot{V}}{V}$

where $V$ is the volume of the chamber and $\alpha_{i n}, \alpha_{\text {out }}$, and $\alpha$ lies between 1 and $\gamma$. Richer and Hurmuzlu (2000) recommended that $\alpha_{i n}$ and $\alpha_{\text {out }}$ should be close to $\gamma$ and 1 respectively and to better describe the thermal behavior of expansion/compression of gas due to piston movement, $\alpha$ should be equal to 1.2.

\section{Testing and characterization}

The tests are carried out on proto-model and concept is demonstrated successfully. Further, system testing for different orifices at pressure $\&$ vent ports and various source pressures is carried out for tuning the mathematical model. Figure 8 gives test set up of proto-model.

The waveforms of inspiration pressure obtained when the relief valve is set at 35 mbar is given in Fig. 9a, b. Further, a set of orifice configurations and source pressure values are fixed for typical $I: E$ ratio and breathing rates. Fine tuning of the system based on initial test results is in progress. The tests with solenoid valves and controller are being conducted.

\section{PRANA}

PRANA ventilator delivers respiratory gases to the patient by automated compression of Ambu bag using an actuator. The system incorporates closed-loop electronic control based on pressure and flow sensors to precisely set the ventilator parameters required by the user.

The actuator for compressing the Ambu bag is driven by a DC motor through a gear train which is unique in this design. The Ambu bag is uniformly compressed at multiple circumferential locations to ensure even compression of the

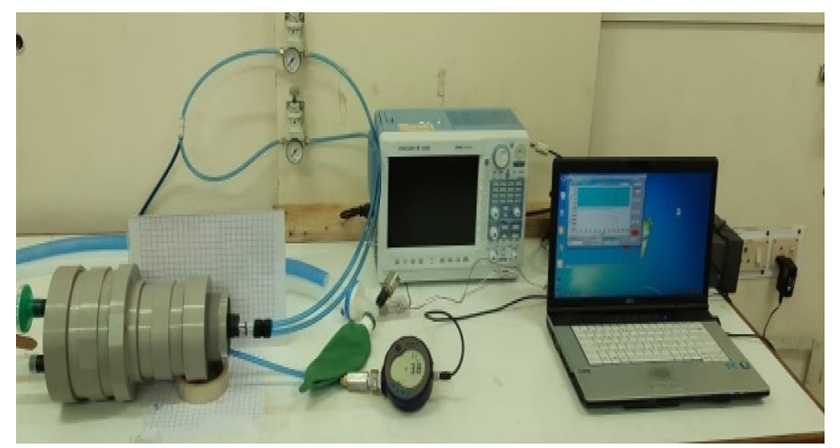

Fig. 8 SVASTA under testing \& validation

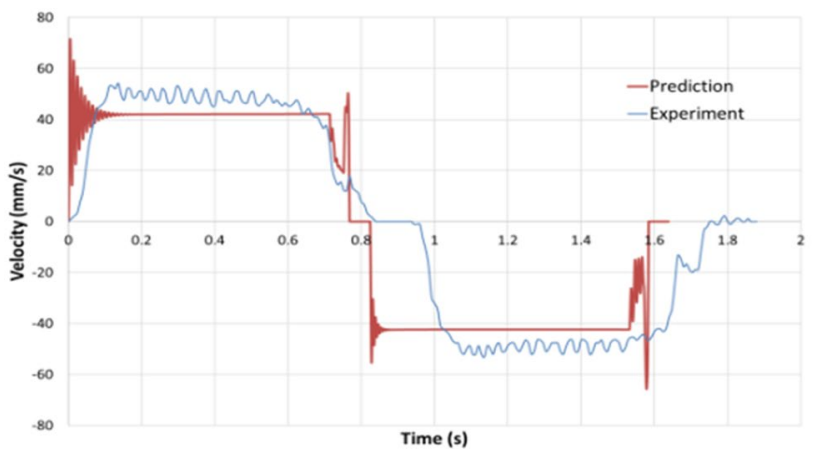

(a)

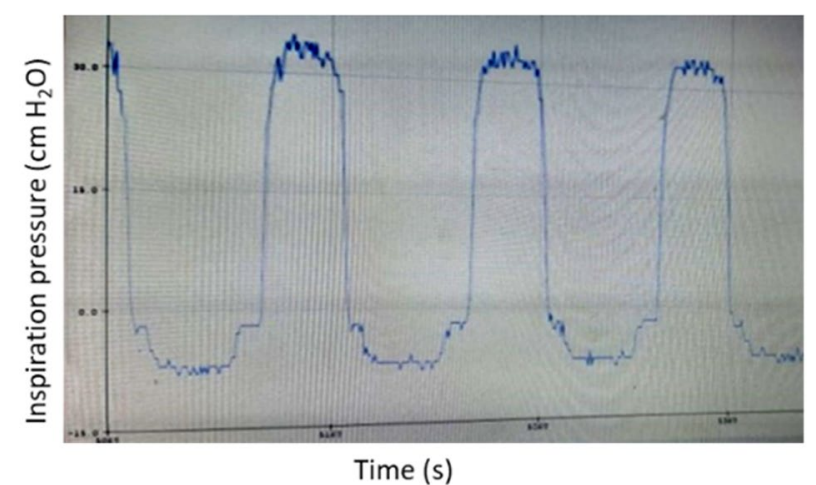

(b)

Fig. 9 a Piston Velocity vs Time obtained through high-speed imaging data and its comparison with prediction (b) Inspiration pressure vs Time obtained through pressure transducer at 35 mbar pressure relief valve setting (display screenshot)

bag. This aids in reducing the stresses on Ambu bag and increasing its life. The ventilator incorporates humidifier for humidifying the respiratory gas delivered to the patient and a suction pump for aiding in expiration.

\section{Configuration}

The configuration of the system is highlighted in Fig. 10. The system has an interface for admitting oxygen and air into the Ambu bag. Outlet of the Ambu bag is attached to the

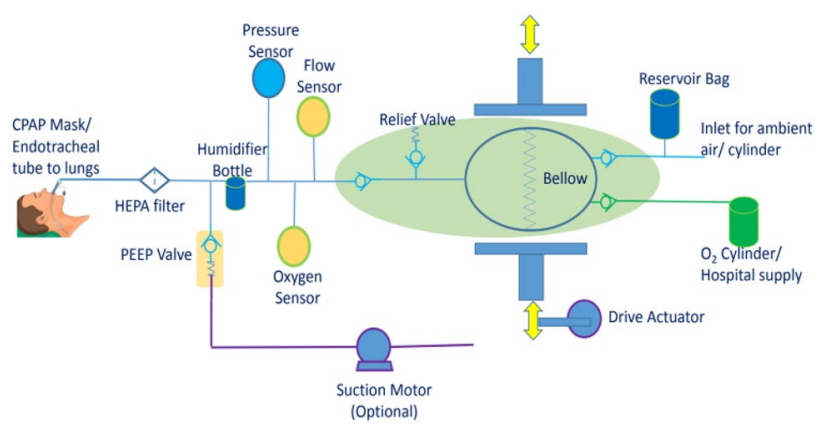

Fig. 10 Configuration of PRANA 
breathing circuit of the patient through a humidifier. Pressure sensors and flow meters are provided in line to sense the inspiratory pressure and inspiratory flow. A pressure relief valve is provided in the line to vent the gases in case the inspiratory pressure exceeds $80 \mathrm{~cm} \mathrm{H}_{2} \mathrm{O}$. Manually settable valve is provided in the expiration line for controlling the PEEP. A suction pump is provided in the line to aid suctioning.

PRANA is a self-contained closed unit consisting of automation mechanism for the BVM unit, sensors, inlet air filtration unit, inspiratory and expiratory line valves and filtration unit for the patient-y-connector, control electronics, display and control unit, battery and power module.

\section{Working}

Oxygen is admitted to the Ambu bag through an oxygen regulator which can be manually set to achieve the required $\mathrm{FiO}_{2}$. Expansion of the Ambu bag takes the respiratory gases into the Ambu bag. Ambu bag is then compressed by the movement of actuator driven by DC motor.

The actuator movement is controlled in closed-loop to achieve the required flow (for VCV) or pressure (for PCV and PSV). The tidal volume delivered is estimated by the controller by integrating the flow. The subsystem of the ventilator realized is shown in Fig. 11.

\section{Mathematical Modeling}

The mathematical model of the ventilator is developed by considering the following sub-systems.

i. Actuator stroke results in a variation of Ambu bag volume. Stroke to volume change is modelled by assuming a curve-fit which can be experimentally derived.

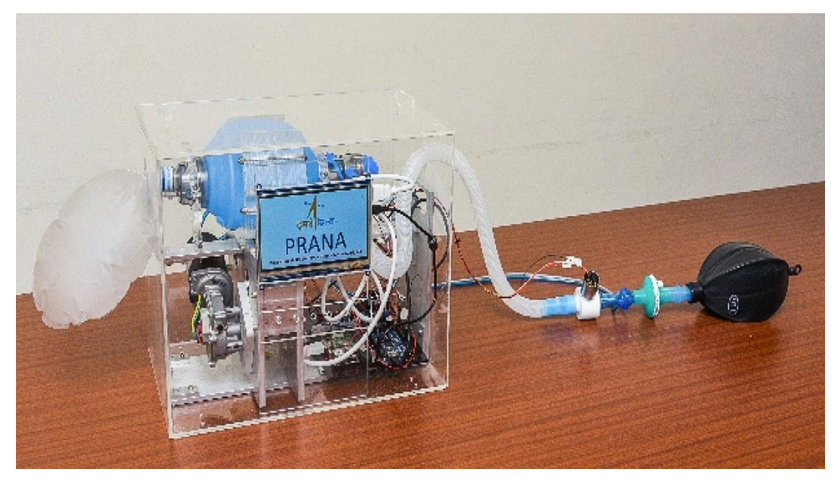

Fig. 11 PRANA system ii. Ambu bag volume to the pressure of the gas in the Ambu bag is derived using gas equations with isothermal assumption.

iii. Pressure in the Ambu bag results inflow from the ventilator to lung. Flow is a function of the lung characteristics. Lung is modelled as a combination of resistance and capacitance to simulate the resistance and compliance of the lung. In this model, lung is represented as a transfer function between Ambu bag pressure and flow from Ambu bag.

iv. A mathematical model of the humidifier is also developed.

In the control system, two measurements are assumedthe pressure of the gas in the Ambu bag and flow from the Ambu bag. Based on these measurements, a pressure control system and volume control system are designed using PID controllers. Detailed mathematical models and corresponding equations are not presented here.

\section{Testing and Characterization}

Ambu bag is typically used for non-invasive ventilation with a mask and does not require much control on the bag-compression. However, the invasive ventilation requires precise control of pressure, flow rate and tidal volume. To achieve this, the ventilator is characterised as following:

i. The Ambu bag is considered as a positive displacement pneumatic source. Hence, the calibration of volume flow with bag compression is the first and the foremost crucial step. The rate of bag compression would also decide the flow rate.

ii. The pressure rise in the ventilator as well as in the lungs results from the restriction of flow in the lungs and its subsequent inflation during the inspiration cycle. Hence it is important to estimate the pressure rise with respect to tidal volume based on lung compliance. This is achieved through a test lung bladder with added external stiffness based on tidal volume. However, the final characterization with human lungs equivalent setup is necessary. It is to be noted that, due to the configuration of the Ambu bag, not all combinations of tidal volume and positive inspiratory pressure requirements would be achievable. Though, the extent of this limitation will be known through the testing of human-equivalent lungs.

iii. Pressure drop and compressibility effect in the ventilator circuits is another important factor. This is characterized through external measurement at the lungs and electronically compensated based on the readings from ventilator sensors. 
The goal of the testing and the characterization is to establish an exact correlation between the parameters set through the ventilator electronics, the same parameters measured by the sensors of the ventilator and the parameters measured through the external sensors in the test lung setup. This would ensure that the patient is getting the same ventilation as set by the doctor and the ventilator display also shows it correctly.

\section{VaU}

Development of pneumatic circuit based option is pursued with the aim of realizing a low-cost state of the art ventilator equivalent to commercially available high-end ventilators. The system is envisaged as a dual-mode ventilator that can work with either medical air/oxygen from the hospital or with ambient air. While working with medical air/oxygen, the respiration parameters are controlled by proportional control valves and while working with ambient air, the parameters are controlled by speed control of blower. The compact design and the capability to work with ambient air allows the device to function not only as ICU ventilator but also as a portable or home ventilation unit. The critical components of the ventilator are indigenously developed with the support of other ISRO Centres.

\section{Configuration of VaU}

The configuration of $\mathrm{VaU}$ is shown in Fig. 12. The system has interfaces for the intake of medical air and medical oxygen from hospital supply and for the intake of ambient air. Electrically operated blower, control valves, sensors, electronics and display is housed in a compact tabletop module.

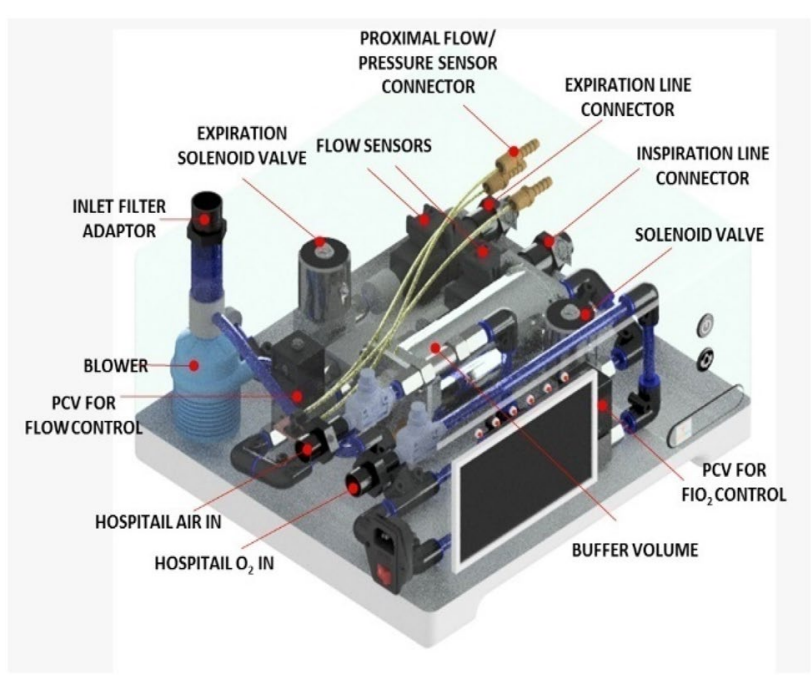

Fig. 12 Configuration of $\mathrm{VaU}$

\section{Working Principle}

The circuit diagram of the system is shown in Fig. 13. While operating with the hospital pneumatic supply, the medical air \& oxygen are passed through suitable filters for the removal of lodged particulates. Needle valves (Metering Ball Valves) provided downstream of the filters allow coarse control over flow rates and serves as safety shut-off in case of exigencies. A proportional control valve in the oxygen line enables electronic control over $\mathrm{FiO}_{2}$. The air and oxygen lines are joined and fed to the inlet of a normally closed solenoid valve. A set of non-return valves provided upstream of the solenoid valve provides backflow protection. The solenoid valve is operated in an on-off control mode to maintain the pressure inside a buffer volume between 400 and 600 mbar(g). The buffer volume minimizes the effects of supply pressure variations on the ventilator function and prevents pressure/flow surges.

The inspiration pressure/flow rate control is achieved using a Proportional Control Valve (PCV2 in Fig. 13) fed by the buffer volume.

In the blower mode, the ambient air is filtered and drawn in by the electrically operated blower through an inlet manifold, which houses a Bacterial Viral Filter. Oxygen is supplied to the inlet manifold through the same circuitry used for the pneumatic option. As in the pneumatic option, a proportional control valve in this line allows control over $\mathrm{FiO}_{2}$. The incoming air-oxygen mixture is compressed by the blower and delivered to the patient. The necessary inspiration pressure/flow control is achieved by the speed control of the blower.

The delivery of the blower and PCV2 is connected to an inspiration manifold through a set of non-return valves. Based on the mode selected, either the blower or PCV2 supplies the required air-oxygen mixture to the manifold, which in-turn passes it on to the inspiration line. The manifold houses a flow sensor and an $\mathrm{FiO}_{2}$ sensor for the measurement of the inspiration flow rate and oxygen concentration respectively. Additionally, it consists of a pressure relief valve for overpressure protection and an

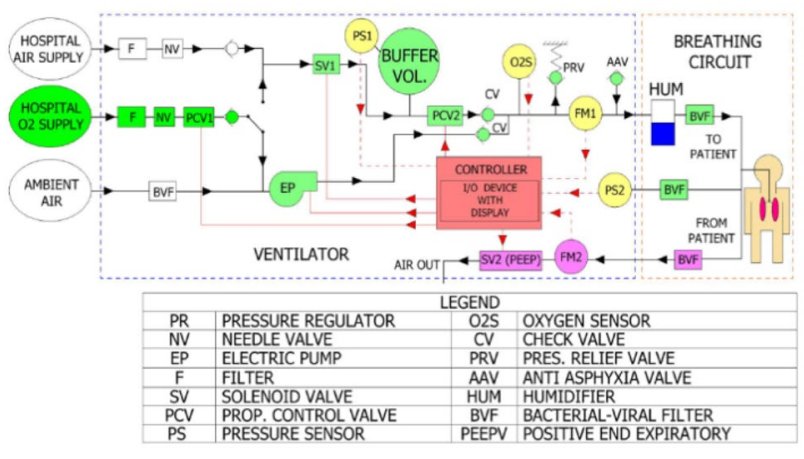

Fig. 13 Circuit diagram of VaU 
anti-asphyxia valve that allows the patient to take spontaneous breaths in the event of a power/equipment failure.

The expired air from the patient is filtered and delivered back to the ventilator through a Bacterial Viral Filter. Inside the ventilator, this air is passed through a flow meter to measure the expiration flow rate and delivered to a NO solenoid valve which is used to maintain the PEEP. The normally open function of the solenoid valve, allows the expired air from the patient to vent freely into the ambient, in the event of a power/equipment failure.

\section{Control Electronics}

A unified Control Electronics (CE) to meet the control requirements of SVASTA \& PRANA is designed and realized. The salient features of the control Electronics are the following:

a. Controller is based on Arduino Mega 2560 commercial off the shelf board for the ease of prototyping and realization

b. Control algorithm based on pressure sensor (for pressure control) and flow sensor (for volume control) in addition to synchronization with the patient inhalation attempt

c. Setting provision provided includes:
i. Mode Control—pressure/volume
ii. Breath per minute
iii. Tidal volume
iv. Inspiration pressure
v. PEEP pressure
vi. Inhalation/Exhalation (I:E) ratio

d. Graphical display interface for parameter display and setting

e. Buzzer and LEDs for emergency alarms and indication

f. Sensor interface
i. Pressure sensor
ii. Flow sensor
iii. Oxygen sensor
iv. Limit switch
v. Feedback potentiometer

g. Control Drive Element

i. SVASTA-Solenoid valve (24 V/0.5A)

ii. PRANA—DC motor $(12 \mathrm{~V} / 1.2 \mathrm{~A})$

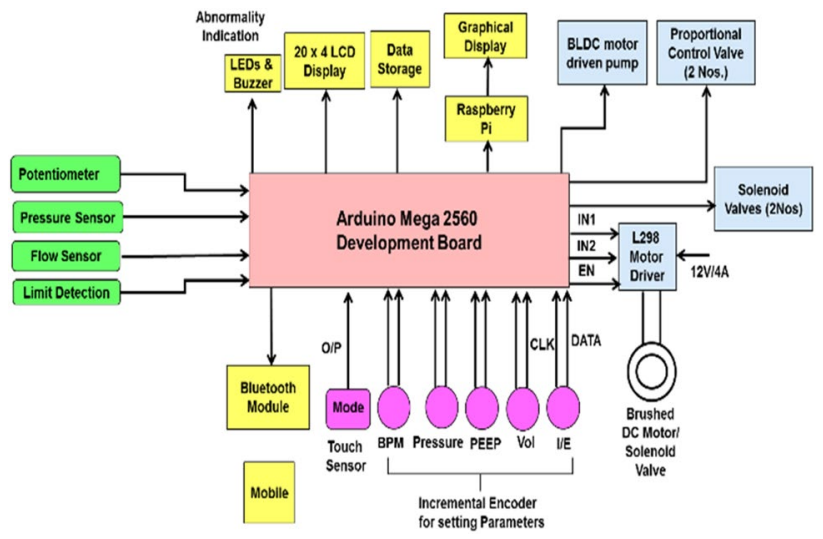

Fig. 14 Control Electronics (CE) Block Diagram

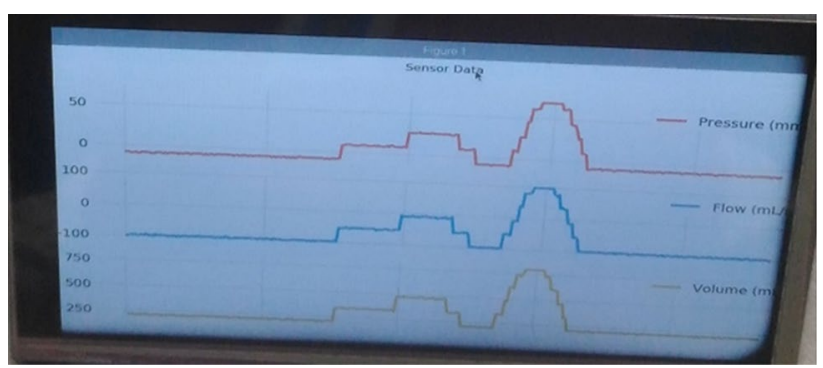

Fig. 15 Display screen interface

\section{Configuration of Control Electronics}

Block diagram of the digital CE is shown in Fig. 14. The CE is based on Arduino Mega 2560 board and is designed considering the need to bring in flexibility in electronics.

$\mathrm{CE}$ has an interface to various sensors and sensor information is used to precisely control the drive elements like motors, solenoid, etc., based on the configuration of control elements used in the ventilator. Additional analog interface is provided for BLDC motor drive and proportional control valve. A display interface is provided for displaying the mode of ventilation and parameters set by the clinician.

To provide the clinician a holistic view of the lung performance, the following parameters are plotted in real time.

- Lung pressure

- Flow rate

- Tidal volume

- Breath per minute

The display screen interface is shown in Fig. 15.

\section{Hardware Elements}

i. Controller 

ii. Sensor interface circuits
iii. Display modules
iv. Parameter setting interfaces
v. Driver interface for motor/solenoid
vi. Battery/Power Interface

\section{Software Elements}

i. Parameter setting module

ii. Emergency indication and alarms module

iii. Parameter display and storage module

It is planned to replace Arduino Mega 2560 board and develop a unified dedicated electronic hardware based on commercial devices meeting all the interface requirements and with added reliability.

\section{Conclusion}

Design features, configuration and working of three ventilator models viz., SVASTA, PRANA, and VaU, are explained. SVASTA offers minimum electricity dependence and has mechanical controls, thus reducing the complexity. However, the extent of precise control on ventilator parameters might not be as effective as in the other models. PRANA is easily realisable and has closed-loop control of critical ventilator parameters. However, it requires inspection and replacement of Ambu bag. VaU offers a more sophisticated and state of the art ventilation system; the critical components of $\mathrm{VaU}$ are being indigenised.

Mathematical modeling of the systems and test results are also highlighted. Configuration and design of control electronics for the ventilators is also detailed. The test and evaluation plan and certification plan is worked out by the quality teams as followed for space systems. VSSC aims to qualify through extensive simulations and certify these prototypes of ventilator variants and transfer the know-how to interested industries for mass production in India.

Acknowledgements Design and Development Team, VSSC: A multidisciplinary team of 30 engineers and technical staff under the overall co-ordination of Technology Development \& Management Group supported by entities of Avionics, Materials and Mechanical, Aerospace Ordnance, Control Guidance \& Simulation and System
Reliability under the overall guidance of Director, VSSC. The design team expresses their gratitude to Chairman, ISRO for the keen interest shown in the development activities, reviews conducted, support and encouragement.

\section{References}

COVID-19 Website of World Health Organisation (WHO). https:// www.who.int/emergencies/diseases/novel-coronavirus-2019. Accessed on 30th April 2020.

Dellaca' RL, Veneroni C, Farre' R (2017) Trends in mechanical ventilation: are we ventilating our patients in the best possible way? Breathe (Sheff) 13(2):84-98. https://doi.org/10.1183/20734 735.007817

Eight Indian Innovations That Could Prove Crucial in Tackling COVID-19, https://www.thebetterindia.com/223156/coronaviru s-COVID19-india-innovation-ventilator-iit-startup-iisc-india n-railways-nor41/. Accessed on 28th April 2020.

Indian Railways manufactures low-cost ventilator prototype for COVID-19 patients in a week's time, https://www.financialexpres s.com/infrastructure/railways/kudos-indian-railways-manufactur es-low-cost-ventilator-prototype-for-COVID-19-patients-in-aweeks-time/1919953/. Accessed on 28th April 2020.

Methods of generating gas flow for mechanical ventilation. https:// derangedphysiology.com/main/cicm-primary-exam/required-readi ng/respiratory-system/Chapter\%2520503/methods-generating -gas-flow. Accessed on 28th April 2020.

Medicines \& Healthcare Products Regulatory Agency(MHRA), Department of health and social care in the United Kingdom (2020). Rapidly Manufactured Ventilator System (RMVS), Document RMVS001-Specification. https://assets.publishing.servi ce.gov.uk/government/uploads/system/uploads/attachment_data/ file/879382/RMVS001_v4.pdf

NASA Develops COVID-19 Prototype Ventilator in 37 Days. https ://www.jpl.nasa.gov/news/news.php?feature $=7646$. Accessed on 28th April 2020.

Richer E, Hurmuzlu Y (2000) A high performance pneumatic force actuator system: part I-Nonlinear mathematical model. J Dyn Sys Meas control 122(3):416-425

World Health Organization (WHO) (2020) Technical specifications for invasive and non-invasive ventilators for COVID 19: Interim Guidance. https://apps.who.int/iris/bitstream/handle/10665/33179 2/WHO-2019-nCoV-Clinical-Ventilator_Specs-2020.1-eng. pdf? sequence $=1 \&$ isAllowed $=y$

Website of Ministry of Health and Family Welfare, Government of India. https://www.mohfw.gov.in/. Accessed on 30th April 2020.

Publisher's Note Springer Nature remains neutral with regard to jurisdictional claims in published maps and institutional affiliations. 